Reprod. Nutr. Dévelop., 1980, 20 (5 A), 1503-1513.

\title{
Essai d'interprétation de l'action de l'eau injectée dans l'artère inguinale sur l'augmentation de pression intramammaire de la brebis
}

\author{
par J. LABUSSIÈRE, Marie-Christine RUIZ, J. F. COMBAUD
}

Laboratoire de la traite, INRA

65, rue de Saint-Brieuc, 35042 Rennes Cedex.

Summary. An attempt to understand why water injected into the udder artery of sheep causes the intramommary pressure to increase.

The increase in intramammary pressure caused by injecting small amounts of water into the udder artery of anesthetized ewes does not seem to be a nervous reaction induced by the stimulation of sensitive mammary receptors : the deflections remain unchanged after complete denervation of the gland (fig. 4) and after the injection of water at different temperatures ( 4 to $37^{\circ} \mathrm{C}$; fig. 1 ) or $\mathrm{pH}$ 's ( 4.6 to 9.8 ; fig. 2 ). Since injecting the same volumes of blood (fig. 3), isotonic $\mathrm{NaCl}$ (fig. 5) or glucose (fig. 6) solutions does not cause the intramammary pressure to increase, water effect would appear to result from a change in blood osmotic pressure.

Among the substances released when the blood corpuscles or platelets are destroyed, kallikrein and histamine do not seem to play a role. These products induce intramammary pressure to rise (fig. 7,9) but latency and duration are longer than with water. Also, their respective inhibitors, aprotinin and phenergan, always block their effect on intramammary pressure but not that of water.

On the other hand, water and bradykinin (fig. 8) produce similar responses, indicating that the latter substance may play a role.

We must consider also the possible action of extra-capillary water transfer, modifving ion flux and myoepithelial cell membrane potential or simply inducing the alveolar cells to swell and the acini to flatten.

This last hypothesis permits a better interpretation of the pressure decreases noted after injection of hypertonic solutions (figs. 5,6 ) since reverse water movements would have the opposite effect.

\section{Introduction.}

Nous avons pu montrer que l'injection de petits volumes d'eau distillée stérile dans l'artère inguinale de brebis Manech anesthésiées provoque une augmentation de pression intramammaire dont l'amplitude est d'autant plus importante que le volume injecté est plus grand (de 0,5 à $8 \mathrm{ml}$ ) et que le temps d'administration est plus court (entre 3,5 et $28 \mathrm{sec}$ ) (Labussière, Ruiz et Combaud, 1979). 
On peut avancer plusieurs hypothèses pour expliquer cette réponse :

1) Le $\mathrm{pH}$ légèrement acide de l'eau distillée et sa température plus basse que la température corporelle pourrait provoquer la stimulation des chemorécepteurs ef thermorécepteurs mammaires et induire un réflexe segmentaire exclusivement nerveux se traduisant par la contraction des canaux galactophores.

2) L'augmentation brutale de pression résultant de l'administration d'un excédent d'eau dans l'artère inguinale pourrait :

- soit stimuler les barorécepteurs du pis et évoquer un réflexe segmentaire analogue à ceux envisagés précédemment ;

- soit comprimer directement les acini et entraîner par simple effet physique l'augmentation de pression observée.

3) Les éléments figurés du sang (érythrocytes, leucocytes, plaquettes) où le tissu mammaire environnant pourraient au contact de l'eau libérer une (ou des) substance possédant un pouvoir contracturant sur les cellules myoépithéliales. Dans ce contexte, on peut envisager la participation de l'histamine dont l'effet sur la mamelle a été mis en évidence par Linzell (1955) mais aussi celle de la kallikréine et de la bradykinine qui provoquent également une augmentation de pression intramammaire (Houvenaghel ef Peeters, 1967 ; Houvenaghel ef al., 1968 ; Houvenaghel et Peeters, 1968 ; Peeters et al., 1972).

L'objet de ce travail est de tester chacune de ces hypothèses.

\section{Matériel et méthodes.}

1. - Préparation des animaux pour les injections. - La mise à jeûn ef l'anesthésie des brebis de race Manech, la pose d'un cathéter dans l'artère inguinale ef l'enregistrement de pression intramammaire sonf effectués selon les méthodes décrites précédemment par Labussière, Ruiz et Combaud (1979).

La dénervation de la mamelle est effectuée selon la technique de Denamur et Martinet (1959).

2. - Caractéristiques des solutions injectées.

a) L'effet éventuel de la température de l'eau distillée $\left(4^{\circ}, 18^{\circ}, 37^{\circ} \mathrm{C}\right)$ et celui du $\mathrm{pH}(4,6 ; 5,3 ; 7,0 ; 8,4 ; 9,8$ obtenus après ajustement avec $\mathrm{NaOH}$ ou $\mathrm{HCl}$, ont été testés par injection intra-inguinale d'un volume de $4 \mathrm{ml}$ en 3,5 ou $7 \mathrm{sec}$.

b) L'influence possible de la masse liquide et de la pression osmotique des substances utilisées a été contrôlée respectivement par l'administration intra-inguinale :

- de volumes identiques (4 ou 8) $\mathrm{ml}$ ) de sang et d'ea.u. Le sang est prélevé dans la veine jugulaire du même animal ef immédiatement réintroduit sans anticoagulants dans l'artère mammaire ;

- de solutions hypotoniques, isotoniques ou hypertoniques de $\mathrm{NaCl}$ ou de glucose dont les concentrations varient de 0 à 45 p. 1000 pour le $\mathrm{NaCl}$ et de 0 à 30 p. 100 pour le glucose.

c) La participation de médiateurs chimiques a été appréciée en comparant leurs effets à celui de $4 \mathrm{ml}$ d'eau en $3,5 \mathrm{sec}$ et des essais d'abolition des réponses ont été 
conduits en utilisant les inhibiteurs spécifiques de ces substances. Les produits utilisés sont les suivants :

- Kinines : La kallikréine qui catalyse la formation de bradykinine est présente à l'état de précurseur (kallikréinogène) dans le sérum sanguin (Trautschold ef Rudel, 1963) et la glande mammaire de vache (Peeters et al., 1976). Sa libération peut être déclenchée par des traumatismes d'origine diverse, dilution du sérum, milieu acide, chaleur, etc... (Trautschold ef Rudel, 1963). Les doses de kallikréine (kalleone lyophylisée Bayer Pharma) variaient entre 160 et 2400 mUK ( $\left.{ }^{1}\right)$ et celles de bradykinine (Sandoz) entre 80 et $800 \mathrm{ng}$.

— Inhibiteurs des kinines : L'étude a été réalisée à l'aide de 10 à 10000 UKI ( $\left.{ }^{2}\right)$ d'un antikallikréique, l'aprotinine (Zymofren, Specia) et de 2 inhibiteurs de la trypsine (9008 et 9253 , Sigma) aux doses respectives de $0,5 \mathrm{mg}$ et $1 \mathrm{mg}$. La trypsine est connue comme étant un activateur de la kallikréine dans la synthèse des bradykinines.

- Histamine : Chez les mammifères, l'histamine sanguine est principalement concentrée dans les globules blancs (70 à 89 p. 100) et peut être libérée au cours de chocs anaphylactiques (Reuse, 1954). Les injections contenaient 0,5 à $1 \mathrm{mg}$ d'Histamine Dihydrochoride (7 250 sigma).

- Antihistaminiques : Parmi la gamme importante des antagonistes de l'histamine, nous avons retenu un inhibiteur compétitif, le Phénergan (Specia) aux doses de 10 à $37,5 \mathrm{mg}$ et un inhibiteur de synthèse, la trifoqualine (Promedica) qui fut administrée par voie orale pendant les 2,5 jours qui précèdent l'enregistrement, à raison de $5 \mathrm{~g}$ matin et soir.

Les kinines, l'histamine et leurs inhibiteurs respectifs (sauf la tritoqualine) ont toutes été diluées dans du sérum physiologique. Elles furent administrées au niveau de l'artère inguinale dans des volumes n'excédant pas $3 \mathrm{ml}$ et poussées par $0,5 \mathrm{ml}$ de sérum physiologique afin de purger le cathéter.

\section{Résultats.}

1. - Eventualité d'un réflexe segmentaire après stimulation de récepteurs mammaires.

a) L'amplitude des réponses ne semble pas modifiée lorsque l'eau est administrée à 4,18 et $37^{\circ} \mathrm{C}$ (fig. 1) ou que son $\mathrm{pH}$ varie de 4,6 à 9,8 (fig. 2) bien que chez

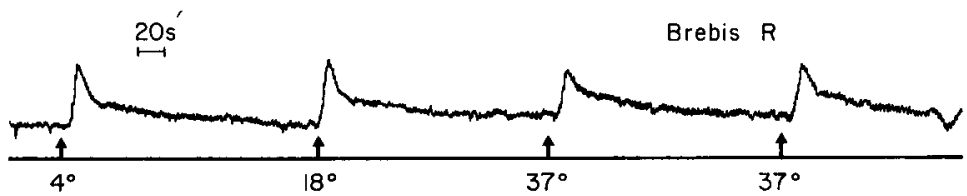

FIG. 1. - Effet de la température de l'eau injectée $(4 \mathrm{ml}$ en $3,5 \mathrm{sec})$ sur la variation de pression intramammaire. Valeurs moyennes et écart-type pour 4 essais : $4{ }^{\circ} \mathrm{C}, 0,25 \mathrm{~mm} / \mathrm{Hg} \pm 0,05 ; 18^{\circ} \mathrm{C}$, $0,23 \mathrm{~mm} / \mathrm{Hg} \pm 0,02 ; 37^{\circ} \mathrm{C}, 0,26 \mathrm{~mm} / \mathrm{Hg} \pm 0,05$.

(1) L'unité kallikréine (UK) est titrée et exprimée en unité inhibitrice de Proteases, telle que 1 unité IP inhibe 1 unité Schwert-Takenaka de Trypsine.

$\left({ }^{2}\right)$ L'unité antikallikréine (UKI) représente la quantité de produit qui, à pH 8 et après 2 h à température ambiante, peut inactiver 50 p. 100 de 2 unités de kallikréine. 
l'animal $C$, il faille noter une légère diminution de la, pression intramammaire au fur et à mesure que le $\mathrm{pH}$ s'accroît.
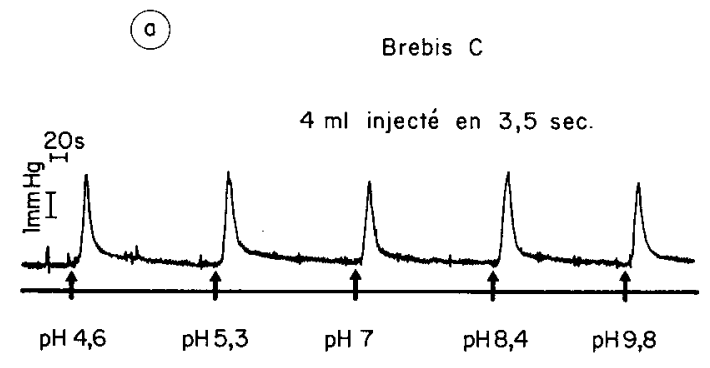

Brebis C

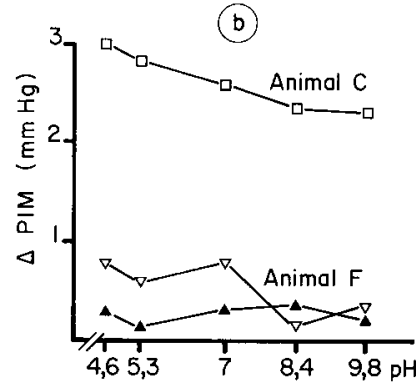

FIG. 2. - Effet du pH de l'eau sur la variation de pression intramammaire : a) Exemple chez la brebis $\mathrm{C}$; b) Courbes moyennes obtenues chez 2 animaux. - $-\square-\square-$ injection de $4 \mathrm{ml}$ en $3,5 \mathrm{sec}$, $\longrightarrow \nabla-\nabla-\nabla-$ injection de $4 \mathrm{ml}$ en $3,5 \mathrm{sec},-\wedge-\Delta-\Delta-$ injection de $4 \mathrm{ml}$ en $7 \mathrm{sec}$.

b) Injecté dans les mêmes conditions que l'eau (même volume, même temps) le sang préalablement prélevé dans la jugulaire ne provoque aucune augmentation de pression intramammaire (fig. 3) lors des 10 essais réalisés sur 4 animaux.

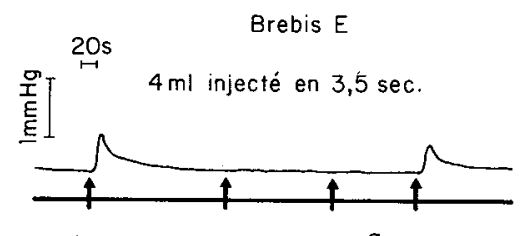

FIG. 3. - Effet de l'injection de sang, de sérum physiologique ef d'eau sur le niveau de pression intramammaire. Sérum $\varnothing$ : sérum physiologique ( 9 p. 1000$)$.

c) La dénervation de la mamelle confirme ces résultats et infirme donc toute hypothèse d'intervention nerveuse puisqu'après cette opération, l'eau induit toujours des réponses d'amplitude sensiblement identique (fig. 4).

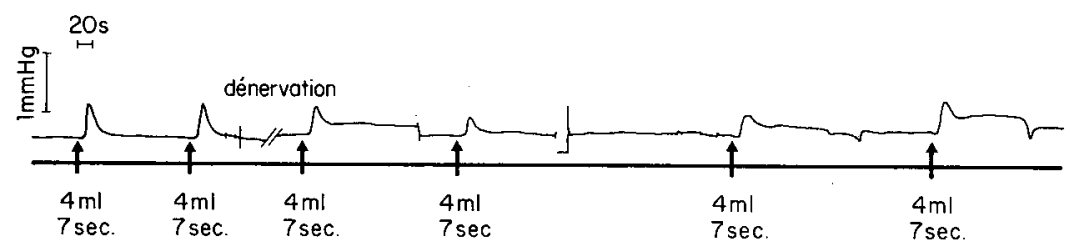

FIG. 4. - Effet de la dénervation de la mamelle sur les modifications du niveau de pression intramammaire dues à l'injection de $4 \mathrm{ml}$ d'eau (animal H).

Comme les injections d'importants volumes de sang $(8 \mathrm{ml})$ ne provoquent aucune augmentation de la Pression Intramammaire, ceci nous conduit à penser que l'action de l'eau pourrait dépendre des modifications de la pression osmotique. 
2. - Effet de l'osmolarité du liquide injecté.

a) Concentration en $\mathrm{NaCl}$. - Nous observons dans tous les cas (4 animaux) la même tendance (fig. 5) :

- Lorsque la concentration augmente de 0 à 9 p. 1000 , l'amplitude des déflections diminue.

- Pour des solutions isotoniques ( 9 p. 1000) ou légèrement hypertoniques, aucune action n'est enregistrée, bien que l'absence de réponse puisse parfois s'observer plus tôt (à partir de 3 p. 1000 ).

- Au-delà, pour des taux très hypertoniques (22 p. 1000,45 p. 1000$)$ les effets observés correspondent à une diminution de plus en plus importante par rapport à la pression de base (Essais effectués uniquement sur 2 brebis).
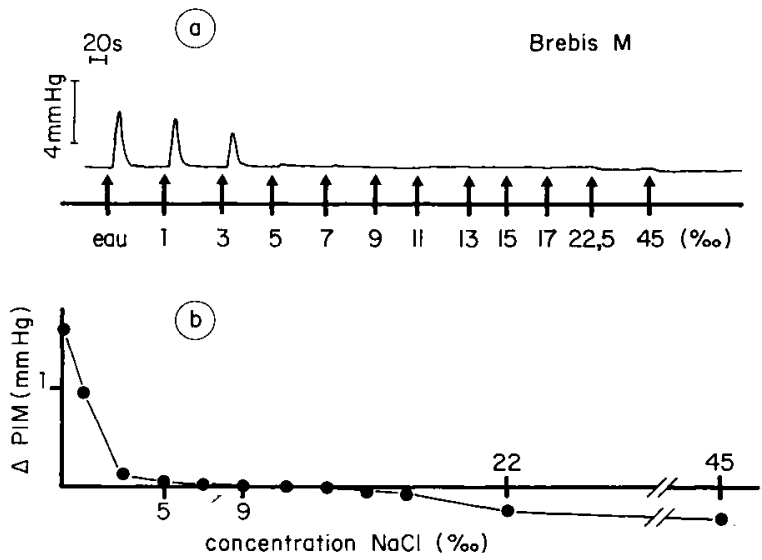

FIG. 5. - Effets de la concentration en $\mathrm{NaCl}$ de l'eau injectée $(4 \mathrm{ml}$ en $3,5 \mathrm{sec}$ ) sur la variation de pression intramammaire. a) Exemple chez la brebis $M$; b) Courbe moyenne obtenue sur 4 animaux.

b) Concentration en glucose. - La tendance observée avec le glucose est très semblable à celle signalée avec le $\mathrm{NaCl}$ (fig. 6). Quand la concentration en sucres :

- augmente de 0 à 5 p. 100, l'amplitude des réponses diminue,

- est isotonique (5 p. 100), celle-ci est nulle,

- devient très hypertonique 15 à 30 p. 100, la pression intramammaire diminue de plus en plus par rapport à la ligne de base.

Ainsi, comme l'injection de solutions isotoniques ( $\mathrm{NaCl}$ ou glucose) n'induit pas de variation de pression intramammaire, on peut donc supposer que la diminution de la pression osmotique du plasma, induite par l'administration d'eau, serait à l'origine des mécanismes responsables des déflexions constatées.

Parmi ces mécanismes, on peut envisager l'intervention d'une substance, soit libérée par les globules sanguins (éventuellement lors de leur éclatement) soit activée au niveau du plasma comme réaction de défense organique au choc provoqué par l'injection. 

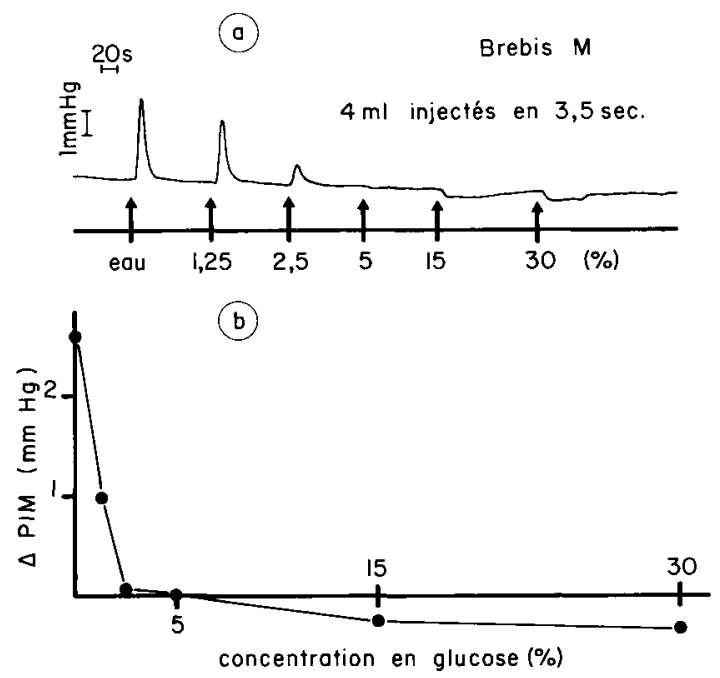

FIG. 6. - Effets de la concentration en glucose de l'eau injectée $(4 \mathrm{ml}$ en $3,5 \mathrm{sec})$ sur la variation de pression intramammaire. a) Exemple chez la brebis $M ; b$ ) Courbe moyenne obtenue sur 2 animaux.

3. - Eventualité de la libération d'une substance déjà identifiée dans la mamelle et pouvant provoquer l'éjection du lait.

a) La kallikréine (6 animaux, 18 essais). - La figure 7 montre les déviations de la PIM obtenues avec la kallikréine. Il apparaît que 800 mUK induisent des déflexions
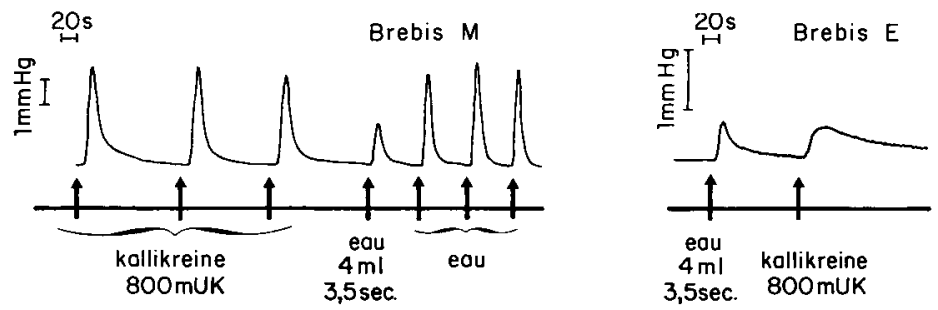

FIG. 7. - Comparaison entre l'effet de l'eau ef celui de la kallikréine sur le niveau de pression intramammaire.

qui peuvent être comparées, chez plusieurs animaux, à celles obtenues avec $4 \mathrm{ml}$ d'eau injectés en $3,5 \mathrm{sec}$, bien qu'à amplitude égale la latence des réponses semble être supérieure de 5 à $8 \mathrm{sec}$ dans le cas de la kallikréine. D'une façon générale, il nous est apparu que l'amplitude et la durée des réponses auraient tendance à augmenter quand la dose de kallikréine s'accroît. C'esi ainsi que chez la brebis $G$, des doses de 160,400, 800 et 1600 mUK correspondent à des amplitudes de 0,23;0,34; 0,$63 ; 0,63 \mathrm{~mm} / \mathrm{Hg}$ et à des durées de 100,120,240 et $260 \mathrm{sec}$. 
b) La bradykinine ( 2 animaux, 8 essais). - Pour les doses comprises entre 80 et $200 \mathrm{ng}$, la bradykinine induit des accroissements de PIM semblables (amplitude, durée, latence) à ceux provoqués par $4 \mathrm{ml}$ d'eau en $3,5 \mathrm{sec}$ (fig. 8).

FIG. 8. - Comparaison entre l'effet de l'eau et celui de la bradykinine sur le niveau de la pression intramammaire.

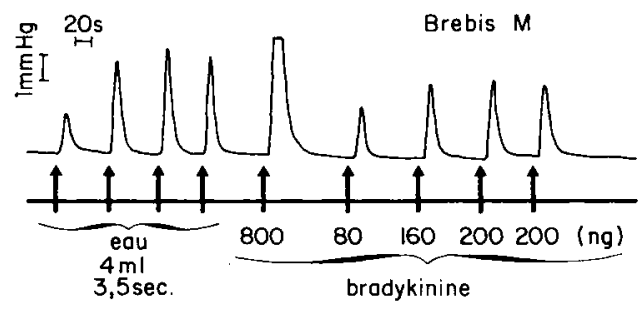

Quand la dose injectée s'élève, l'amplitude el la durée des réponses ont tendance à augmenter. C'est ainsi que chez la brebis $M$, des doses de 80, 160, 200, $800 \mathrm{ng}$ correspondent à des amplitudes de 1,$90 ; 2,90 ; 3,00 ; 6,50 \mathrm{mmHg}$ et à des durées de $36,44,56$ et $60 \mathrm{sec}$.

c) L'histamine (4 animaux, 13 essais). - La figure 9 indique que l'aspect des variations de pression intramammaire provoqués par l'histamine est différent de celui obtenu après injection d'eau.
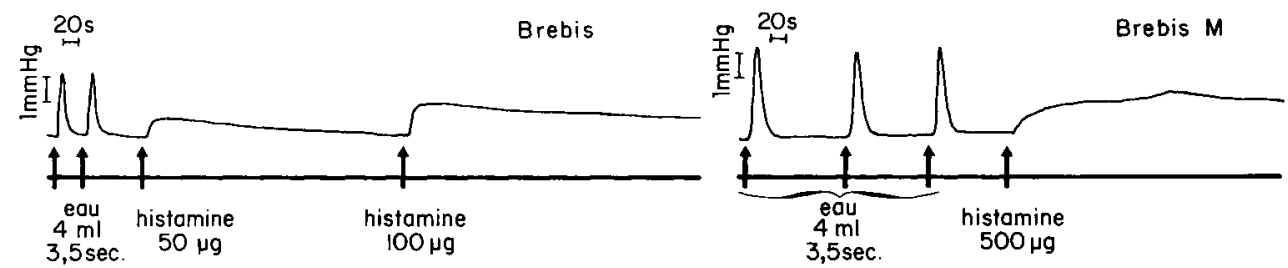

FIG. 9. - Comparaison entre l'effet de l'eau et celui de l'histamine sur le niveau de pression intramammaire.

La durée des réponses est plus longue et il esł parfois même impossible d'observer le retour du tracé à la pression de base.

L'amplitude observée avec $500 \mu \bar{g}$ d'histamine reste inférieure à celle enregistrée avec $4 \mathrm{ml}$ d'eau administrée en $3,5 \mathrm{sec}$. Elle semble toutefois augmenter lorsque la dose d'histamine s'accroît.

d) Bloqueurs des kinines. - L'aprotinine ne provoque aucune augmentation de pression intramammaire mais son administration (10 à $10000 \mathrm{UKI}$ ) bloque toujours

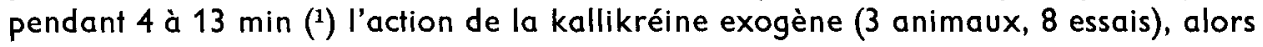
qu'elle n'empêche jamais une augmentation de PIM provoquée par l'eau puisque les

(1) Au-delà de ce délai, les réponses à la kallikréine réapparaissent. 
réponses obtenues, alors, ont une amplitude, une durée et une latence semblables à celles des injections témoins (fig. 10).

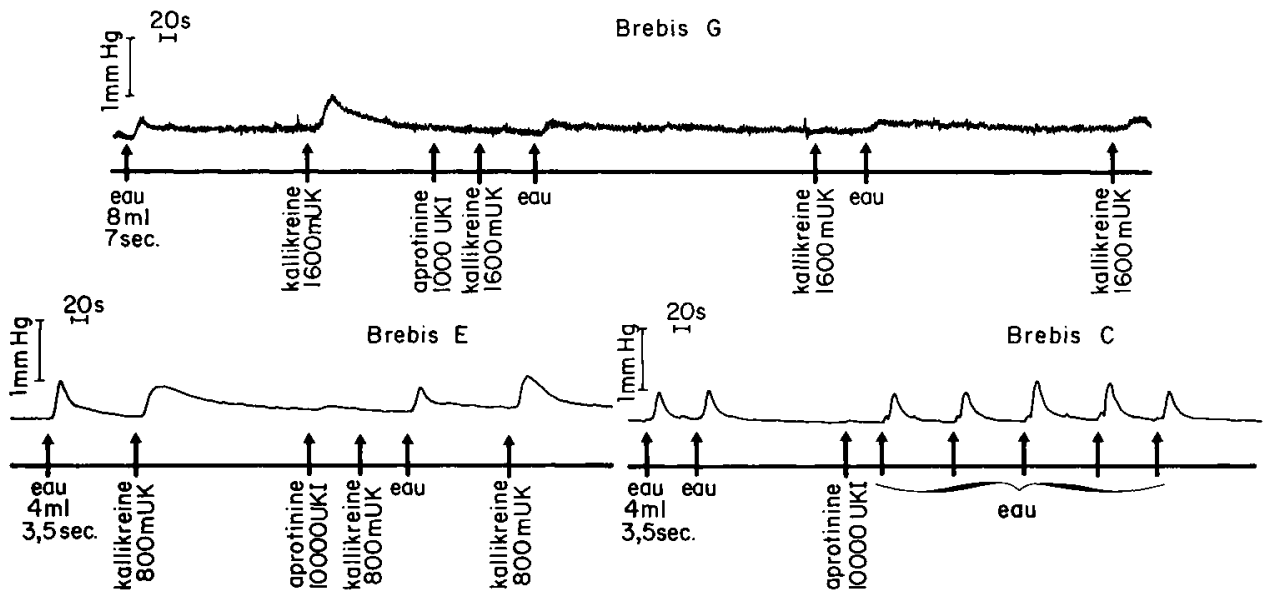

FIG. 10. - Effet de l'aprotinine sur l'augmentation du niveau de pression intramammaire provoquée par l'eau ef la kallikréine.

Les inhibiteurs de la trypsine $9008(1000 \mu \mathrm{g})$ ef $9253(500 \mu \mathrm{g})$ ne suppriment jamais les variations de pression induites par l'eau et la kallikréine.

e) Antihistaminiques. - Le phénergan induit lui-même une augmentation de pression intramammaire mais bloque de manière durable l'action de l'histamine exogène sans supprimer les déflexions provoquées par l'eau (fig. 11).

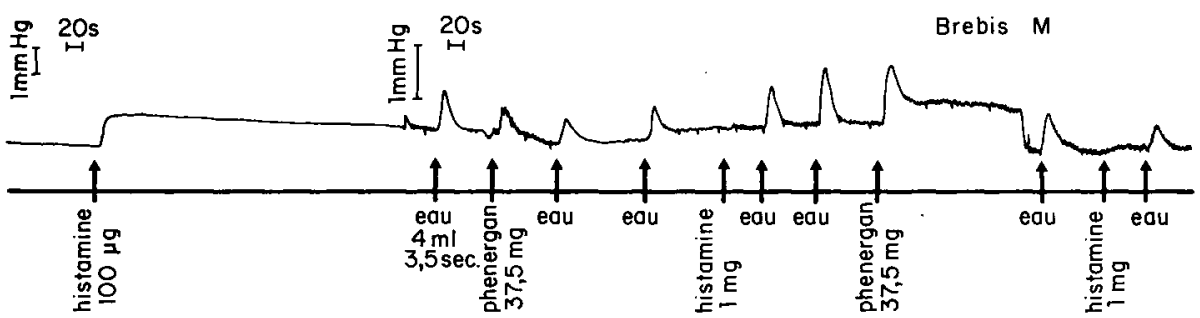

FIG. 11. - Effet du phénergan sur l'augmentation de pression intramammaire provoquée par l'eau et par l'histamine.

Les caractéristiques de ces déflexions ne semblent pas ou peu modifiées chez 4 des 5 brebis étudiées.

Par contre, chez la dernière, les injections d'eau qui ont fait suite aux traitements par l'histamine ef le phénergan ont entraîné 2 réponses, 10 à 15 fois supérieures à toutes celles obtenues jusqu'alors.

Bien que nous ne soyons pas en mesure d'interpréter ces observations, il ne semble pas que l'histamine soit impliquée dans les variations de pression intramammaire 
provoquées habituellement par l'eau puisqu'après 2,5 jours de traitement oral avec $5 \mathrm{~g}$ (matin et soir) de tritoqualine (inhibiteur de synthèse de l'histamine) nous n'avons pu observer leur abolition.

\section{Discussion. Conclusions.}

Les augmentations de pression intramammaire constatées lors de l'injection d'eau distillée dans l'artère inguinale (Labussière, Ruiz ef Combaud, 1979) ne semblent pas dépendre d'un contrôle nerveux induit par la stimulation des récepteurs sensitifs mammaires puisqu'elles ne sont pas modifiées après dénervation totale de la mamelle et lorsque la température de l'eau varie de 4 à $37^{\circ} \mathrm{C}$ ou que son $\mathrm{pH}$ passe de 4,6 à 9,8 .

Comme l'injection d'un volume équivalent de sang n'entraîne aucune augmentation de pression intramammaire et qu'il en est de même après administration de solution itotonique du $\mathrm{NaCl}$ ou de glucose, il nous faut donc supposer que l'action de l'eau résulte d'une modification de la pression osmotique du sang. A cet égard, deux hypothèses peuvent être envisagées.

\section{1. - Première hypothèse.}

Une pression osmotique faible entraînerait la libération par les organites sanguins d'une ou plusieurs substances susceptibles de provoquer la contraction des cellules myoépithéliales. On sait, en effet, que l'injection d'eau dans le sang produit une hémolyse locale et nous pourrions donc admettre que la substance libérée par les globules ou les plaquettes lors de leur éclatement serait responsable de l'accroissement de pression intramammaire.

Parmi les trois substances envisagées, qui ont précédemment été identifiées dans la mamelle (Linzell, 1955 ; Houvenaghel et Peeters, 1968 ; Peeters et al., 1972 ; Peeters et al., 1976) la kallikréine et l'histamine ne sembleraient pas être impliquées dans les modalités d'action de l'eau. Comme l'avaient déjà montré Houvenaghel ef Peeters (1968), Peeters et al. (1972) et Linzell (1955), ces produits induisent bien des élévations de la PIM mais leurs latences ou leurs durées ( ${ }^{1}$ ) sont plus longues que celles obtenues avec l'eau. De plus, leurs inhibiteurs respectifs : l'aprotinine et le phénergan bloquent toujours leur action sur la PIM, sans jamais supprimer l'effet de l'eau.

Par contre, la similitude des réponses, obtenues entre l'eau ef la bradykinine nous conduit à ne pas écarter l'intervention éventuelle de cette dernière substance lors de l'injection d'eau. Ceci reste toutefois à vérifier à l'aide de bloqueurs spécifiques et de techniques biochimiques de séparation et d'identification.

Enfin il convient aussi de ne pas exclure la participation de nombreuses autres substances (adrénaline, acétylcholine, substance $P$, prosłaglandines, 5-hydroxytryptamine) qui peuvent être libérées au niveau vasculaire sous l'effet de chocs ou d'agressions diverses et qui sont connues pour provoquer une éjection du lait (Linzell, 1955). Il importera donc également de contrôler leur contribution bien que pour les prosta-

(1) Surtout pour l'histamine. 
glandines de type $F$ nous n'ayons pas été en mesure jusqu'à maintenant de mettre en évidence leur action directe sur le myoépithélium (Labussière, Guégan et Combaud, 1980).

\section{2. - Deuxième hypothèse.}

A la suite de l'injection d'eau, une partie de celle-ci serait transférée des capillaires vers le milieu extracellulaire puis vers les cellules suivant le schéma de Starling (1909). Cette entrée de liquide au niveau des compartiments intra ef extracellulaire est susceptible d'intervenir de deux façons.

a) Elle pourrait agir sur l'équilibre des flux d'électrolytes ( $\mathrm{Ca}, \mathrm{Na}, \mathrm{K}$ ) entrant et sortant des cellules myoépithéliales ; le changement de la répartition des ions entre le milieu interne et externe de ces cellules serait responsable de la modification de potentiels de membrane et de la contraction qui pourrait en résulter. L'influence de l'environnement ionique sur l'excitabilité du tissu contractile mammaire a été démontrée par Moore et Zarrow (1965), Somlyo, Woo et Somlyo (1966), Lawson et Sch midt (1972).

b) Elle provoquerait le gonflement des tissus épithéliaux et par voie de conséquence une augmentation de pression du lait dans la lumière des acini.

Inversement, une solution hypertonique provoquerait le mouvement d'eau contraire (des cellules sécrétrices vers les capillaires) qui induirait une diminution de la pression des acini sur la lumière alvéolaire et une remontée du lait citernal Cette hypothèse aurait l'avantage d'expliquer les diminutions de pression intramammaire PIM obtenues avec l'injection de solutions hypertoniques de $\mathrm{NaCl}$ et de glucose.

Il nous est actuellement difficile de choisir entre les différentes hypothèses qui viennent d'être envisagées et il serait prématuré de conclure sur l'intérêt de ces observations purement expérimentales, en particulier sur le déroulement des mécanismes qui président à l'évacuation du lait dans les conditions physiologiques naturelles. II semble, en effet, peu probable que la réduction du taux de métabolites entre le sang artériel et veineux soit en mesure d'entraîner la descente du lait alvéolaire dans la citerne entre les traites ou les tétées.

Ce travail préliminaire mérite donc d'être poursuivi par la comparaison des effets de l'eau avec ceux des autres agents pharmacologiques susceptibles de provoquer une augmentation de pression intramammaire. Parallèlement à une analyse hématologique détaillée, il apparaît également souhaitable d'essayer d'isoler ces substances dans le sang mammaire et de vérifier que l'injection de tout ou partie de celui-ci peut induire des réponses équivalentes sur d'autres sujets.

Enfin les essais de modification de l'osmolarité du sang et des liquides interstitiels devront être complétés par l'administration in vivo et in vitro des solutions salines diversement concentrées (simples ou combinées) qui n'ont pas encore été utilisées au cours de cette éfude (calcium, potassium, magnésium, etc...). 


\section{Références}

DENAMUR R., MARTINET J., 1959. Le rôle du système nerveux de la glande mammaire dans l'entretien de la lactation. Arch. Sci. physiol., 13, 271-352.

HOUVENAGHEL A., PEETERS G., 1967. Influence de la bradykinine sur l'éjection du lait chez la brebis et la chèvre. Arch. int. Pharmacodyn., 167, 483-485.

HOUVENAGHEL A., PEETERS G., 1968. Influence de la bradykinine sur l'éjection du lait chez la brebis et la chèvre. Arch. int. Physiol. Biochem., 76, 647-657.

HOUVENAGHEL A., PEETERS G., VANDAELE G., DJORDJEVIC N., 1968. Influence des kinines du plasma et des kallikréines sur l'éjection du lait chez les ruminants. Arch. int. Phys. Bioch., 76, 658-679.

LABUSSIÈRE J., RUIZ M. C., COMBAUD J. F., 1979. Rise in the intrammary pressure of the ewe after injection of water into the inguinal artery. Ann. Biol. anim. Bioch. Biophys., 19, 1843. 1848.

LABUSSIĖRE J., GUEGAN J., COMBAUD J. F., 1980. Effet des prostaglandines sur l'éjection du lait de la vache et de la brebis (à paraître).

LAWSON D. M., SCHMIDT G. H., 1972. Role of cations in milk-ejecting action of oxytocin in rat mammary tissue. Am. J. Physiol., 222, 444-449.

LINZELL J., 1955. Some observations on the contractile tissue of the mammary gland. J. Physiol. London, 130, 257-267.

MOORE R. D., ZARROW M. X., 1965. Contraction of the rabbit mammary strip in vitro in response to oxytocin. Acta endocrinol., 48, 186-198.

PEETERS G., HOUVENAGHEL A., VERBEKE R., REYNAERT R., 1972. Effects of bradykinin and kallikrenine injected into the udder artery of sheep and goats. Arch. int. Pharmacodyn., 198, 397-414.

PEETERS G., VERBEKE R., HOUVENAGHEL A., REYNAERT R., 1976. Isolation of kallikrein from mammary gland of cows. J. exp. Physiol., 61, 1-14.

REUSE J. J., 1954. Histamine, histamino libérateurs ef ontihistaminiques. Masson et Cie Ed., 195-225. STARLING E. H., 1909. The fluid of the body. Keener Ed. Chicago.

SOMLYO A. V., WOO C. Y., SOMLYO A. P., 1966. Effects of magnesium on posterior pifuitary hormone action on vascular smooth muscle. Am. J. Physiol., 210, 705-714.

TRAUTSCHOLD I., RUDEL G., 1963. Données sur l'inhibiteur de la frypsine et de la kallikréine. Arzneim ittelforsch. (Drug Res.), 16, 1507-1515. 